\title{
Islands in the sky: the impact of Pleistocene climate cycles on biodiversity Allan J Baker
}

Address: Department of Natural History, Royal Ontario Museum, Toronto, Ontario, Canada M5S 2C6, and Department of Ecology and Evolutionary Biology, University of Toronto, Toronto, Ontario, Canada M5S 3B2. Email: allanb@rom.on.ca

Published: 3 November 2008

Journal of Biology 2008, 7:32 (doi:10.1 186/jbiol90)

The electronic version of this article is the complete one and can be found online at http://jbiol.com/content/7/9/32

(c) 2008 BioMed Central Ltd

\begin{abstract}
Genetic studies of organisms based on coalescent modeling and paleoenvironmental data, including a new study in BMC Biology of Mexican jays in the sky islands of Arizona and northern Mexico, show that populations differentiated in multiple refugia during and after glacial cycles.
\end{abstract}

The general cooling of the world's climate that began in the Tertiary and culminated in the Pleistocene glacial cycles from about 2.4 million years ago attracted the attention of evolutionary biologists because of its possible effect in changing species distributions, and thus on the speciation of organisms. The role of these climatic fluctuations on speciation has been much debated. At one end of the debate, some researchers argued that the cooling suppressed or slowed speciation, as leading-edge waves of species populations repeatedly colonized deglaciated regions in the interglacial periods $[1,2]$. This form of repeated colonization of genetically similar individuals from the same source populations can prevent genetic differentiation required for speciation. Others thought that the cooling, and the barriers of ice that divided up populations, increased the rate of speciation; in an extreme example of this view, Ernst Mayr wrote in his classic 1970 book [3] that "Evolutionists agree on the overwhelming importance of Pleistocene barriers in the speciation of temperate zone animals".

Data from studies of North American songbirds have been useful in showing which of these two views is correct. As late as 1999, it was thought that species and species complexes of North American songbirds diverged in the late
Pleistocene, which would support the view that climate cooling increased the rate of speciation [4]. This was, however, refuted convincingly by mitochondrial DNA data that suggested that the emergence of new songbird species appeared repeatedly over the past 5 million years, which would mean a much smaller role for climate cooling in speciation [5].

The current consensus is that some species of songbirds originated earlier in the Pleistocene, before the glaciations started [5-7]. It is also generally agreed that strong population structure has evolved in songbirds and in many other organisms [5-8]. When many genetic differences accumulate in different populations, this structures species into isolates that can be a precursor to speciation. However, there is some evidence that songbird speciation might have been completed during late glacial advances by repeated bouts of geographical isolation, as shown by the fact that divergence times estimated with a molecular clock in superspecies complexes of boreal (boreal forest) superspecies of North American birds date to the Pleistocene [9]. These complexes are groups of very similar emergent species with adjacent distributions that are restricted to boreal forests that were glaciated in the Pleistocene. 


\section{Uncertainty in inferences of glacial refugia}

Although there is compelling evidence that ancestral source populations can differ genetically, there is uncertainty about whether isolation of populations that survived and differentiated in glaciated areas called glacial refugia is required to explain genetic differentiation in extant populations $[7,10]$. Furthermore, inference of the number of these refugia and the timing of isolation of populations has, until recently, depended on the construction of gene trees, assumptions about whether these trees reflect population trees, calibrations of molecular clocks and mutation rates of the genes being studied. All these components have uncertainties inherent in their estimates. Innovative new studies have, however, begun to address these uncertainties with exciting insights into the impact of Pleistocene climatic cycles on population differentiation and, potentially, on speciation [10-14].

Evidence for divergence within species complexes of songbirds in both the Pleistocene period and postglacially has been presented in recent studies $[13,14]$. The yellowrumped warbler complex comprises two North American migratory subspecies, the myrtle warbler (Dendroica coronata coronata and Audubon's warbler (D. c. auduboni), previously thought to be separate species, and two largely sedentary (non-migrating) forms from Mexico (D. c. nigrifrons) and Guatemala (D. c. goldmani). The North American forms breed in higher-latitude locations than the Mesoamerican forms, locations that were glaciated in the past. The North American forms hybridize with the Mesoamerican forms only in a narrow hybrid zone in British Columbia and Alberta, but they migrate and overlap with the Mesoamerican forms in winter.

Phylogenetic analyses of three mitochondrial DNA genes using Bayesian methods that account for phylogenetic uncertainty have shown, surprisingly, that the two Mesoamerican forms are reciprocally monophyletic, that is, that they each form a monophyletic group that is phylogenetically separated from the other, whereas the North American forms have high levels of shared ancestral polymorphisms [13]. Assuming a mutation rate of $2 \%$ per million years, a coalescent approach yielded population divergence times of about 400,000 years ago between Mesoamerican and North American forms and 16,000 years ago between the two North American forms. Coalescent theory is a population genetics model that traces all the alleles of a gene in a population sample to one ancestral copy shared by all members of the population, which is called the most recent common ancestor (MRCA). By applying a mutation rate for the gene it is possible to obtain the time in years when the MRCA existed, which approximates when the forms diverged unless they continued to exchange

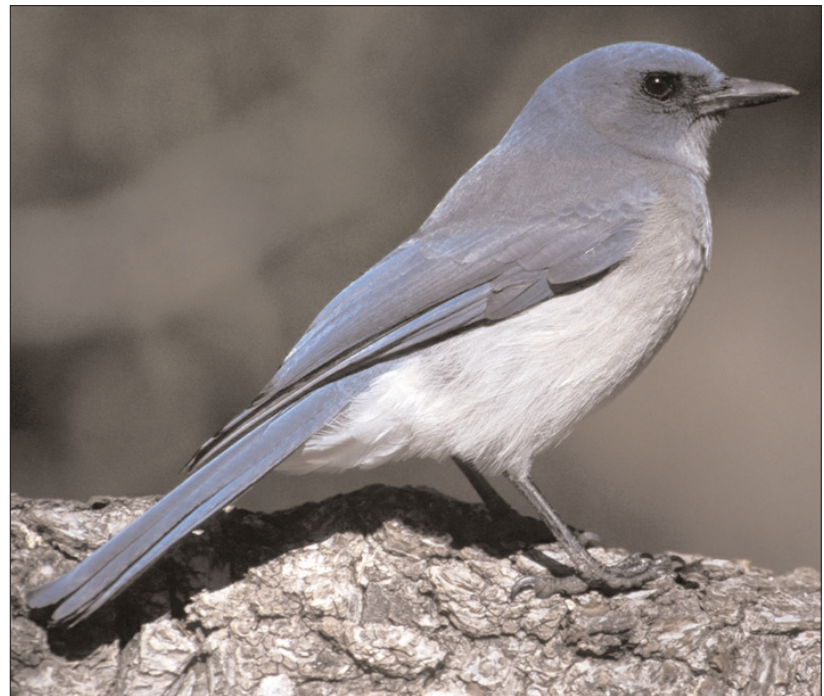

\section{Figure I}

The Mexican jay is a sedentary species found in pine-oak woodlands in the sky islands in the southwestern USA and northern Mexico. Different populations have differentiated genetically within the last 10,000 years. Photo by TJ Ulrich with permission from Visual Resources for Ornithology, the Academy of Natural Sciences, Philadelphia, PA.

alleles for some time after they separated. However, when dated with a wide range of gene-specific mutation rates, the uncertainty in dates was revealed, ranging up to 1.9 million years ago between migratory and sedentary forms and up to 41,000 years ago between migratory forms.

\section{Coupling paleoenvironmental and genetic modeling}

With such imprecision in estimating divergence times, it is difficult to test hypotheses of postglacial population differentiation or rapid speciation using genetic data alone. Now, however, fossil paleoecological data have emerged that can provide an independent timeframe for recent postglacial genetic divergence. McCormack et al. in a recent study in BMC Biology [14] capitalized on populations of Mexican jays (Aphelocoma ultramarina) in the 'sky islands' - isolated mountain niches - of southwestern USA and northern Mexico; these birds are ecologically tied to pine-oak woodlands (Figure 1). Fossilized plant material in the garbage collected in the middens of packrats (Neotoma spp.) showed that the sky islands were connected by continuous woodlands 18,000 years ago, at the last glacial maximum, but as climate warmed in the past 9,000 years the woodlands have been driven to higher elevations and have been displaced by grassland and desert at lower elevations. The authors [14] therefore predicted that populations of jays should share common alleles from the ancestral population, 
but that each population should have a suite of 'private' alleles that has accumulated by mutation in the postglacial period. That is exactly what they found in judiciously chosen mitochondrial and nuclear loci with high mutation rates.

McCormack et al. then subjected the genetic data for selected population pairs to a multilocus coalescent analysis to estimate the time of population divergence and obtained confirmation of postglacial differentiation in the past 10,000 years or less, on the basis of the $90 \%$ highest posterior density distributions. By fitting a model of population splitting to explain the genetic data it is possible to generate a large number of possible estimates of a parameter, which forms the posterior density distribution of parameters, such as population divergence time. This method also takes into account the uncertainties in the simulation process. Additional corroboration of the coalescent estimates was obtained from genetic distances corrected for within-species polymorphism, with the exception that divergence times in the western sky islands in the Arizona 'archipelago' were found using this method to range up to 81,000 years ago. The general message that emerges from this excellent study [14] is that detection of postglacial divergence requires large sample sizes to detect private alleles arising from new mutations and to reduce stochasticity in the coalescent process modeled with or without migration.

\section{Ecological-niche modeling and statistical testing of hypotheses}

Other exciting developments that are helping us to understand the impact of climate-induced shifts in the Pleistocene on distribution of populations, and thus on speciation, include the use of ecological-niche modeling to predict past geographic distributions of ancestral source populations. This innovative approach provides the tools for statistical testing of hypotheses about multiple refugia by integrating inferred past distributions with coalescent-based genetic models [10-12]. Again, these studies are using the multiple replicates provided by different sky-island populations in North America and include a plant-insect herbivore association [12] and montane grasshoppers [10,11].

Cutting-edge research from the Knowles laboratory at the University of Michigan [10,11] using ecological-niche modeling has provided a reconstructed historical distribution of the flightless montane grasshopper (Melanoplus marshalli), revealing that, during glacial maxima, sky-island grasshopper populations in Colorado and Utah must have been displaced to lower refugial areas nearby. By coupling this approach with genetic modeling, the authors were able to test statistically whether the grasshoppers survived in a single ancestral refugial population or multiple refugial populations. Genetic modeling in a coalescent framework not only accounts for the stochastic effects of genetic drift in patterns of population divergence, but by simulating DNA sequences it also incorporates the effect of mutational variance. This makes it possible to use the amount of lineage sorting in extant populations, as measured by the number of deep coalescents in gene trees, to test whether the amount of discord between the sequence data and a two-refugia model is significantly lower than expected under a single refugium model. Recolonization from multiple or single refugia in interglacials could therefore possibly explain why populations of grasshoppers have either evolved strong geographic structure or have speciated, whereas others have differentiated relatively little.

By bringing more biological realism from the natural history of organisms into ecological and genetic modeling of population divergence, the impact of glacial cycles on current biodiversity is being revealed in increasing detail. An interesting aspect of several of these studies is that they often choose to sequence the mitochondrial cytochrome oxidase gene (COI), sometimes in tandem with multiple nuclear genes. $\mathrm{COI}$ is used because it has sufficient variable sites in the part of the gene used in DNA barcoding studies to provide sufficient resolution for coalescent analysis. This point is made clearly in the Mexican jay study [14] and is a straightforward prediction of the faster coalescent times and resolving power of mitochondrial genes [15]. Although the current emphasis in detecting very recent (postglacial) population divergence is on analysis of increasing numbers of nuclear sequences to reduce variance across loci, it seems unwise not to combine these with one or more faster evolving mitochondrial genes, as was done so effectively with the montane grasshoppers [10]. Ultimately, such a unified approach is likely to help delimit species genetically and to connect the processes of population divergence and species recognition in a more rigorous way.

\section{Acknowledgements}

I thank Visual Resources for Ornithology for permission to reproduce Figure I.

\section{References}

I. Coope GR: Late Cenozoic fossil coleoptera. Ann Rev Ecol Syst 1979, 10:247-267.

2. Hewitt GM: The genetic legacy of the Quarternary ice ages. Nature 2000, 405:907-913.

3. Mayr E: Populations, Species and Evolution. Cambridge, USA: Harvard University Press; 1970.

4. Mengel RM: The probable history of species formation in some northern wood warblers. Living Bird 1964, 3:9-43. 
5. Klicka J, Zink RM: The importance of recent ice ages in speciation: a failed paradigm. Science 1997, 277:1666-1669.

6. Avise JC, Walker D: Pleistocene phylogeographic effects on avian populations and the speciation process. Proc Biol Sci 1998, 265:457-463.

7. Wenink PW, Baker AJ, Rosner H, Tilanius, MG]: Global mitochondrial DNA phylogeography of Holarctic breeding dunlins (Calidris alpina). Evolution 1996, 50:3 I8-330.

8. Knowles LL: Tests of Pleistocene speciation in montane grasshoppers (genus Melanoplus) from the sky islands of western North America. Evolution 2000, 54: I337-I348.

9. Weir JT, Schluter D: Ice sheets promote speciation in boreal birds. Proc Biol Sci 2004, 27I: I88।-1887.

10. Knowles LL, Carstens BC, Keat ML: Coupling genetic and ecologicalniche models to examine how past population distributions contribute to divergence. Curr Biol 2007, 17:940-946.

II. Knowles LL: Genealogical portraits of speciation in montane grasshoppers (genus Melanloplus) from the sky islands of the Rocky Mountains. Proc Biol Sci 200I, 268:3 I 9-324.

12. DeChaine EG, Martin AP: Using coalescent simulations to test the impact of Quarternary climate cycles on divergence in an alpine plant-insect association. Evolution 2006, 60:1004-1013.

13. Milá B, Smith TB, Wayne RK: Speciation and rapid phenotypic differentiation in the yellow-rumped warbler Dendroica coronata complex. Mol Ecol 2007, 16:159-173.

14. McCormack JE, Bowen BS, Smith TB: Integrating paleoecology and genetics of bird populations in two sky island archipelagos. BMC Biol 2008, 6:28.

15. Zink RM, Barrowclough GF: Mitochondrial DNA under siege in avian phylogeography. Mol Ecol 2008, I7:2107-2I 21 . 